\title{
A TEST COMPLEX FOR GORENSTEINNESS
}

\author{
LARS WINTHER CHRISTENSEN AND OANA VELICHE
}

(Communicated by Bernd Ulrich)

\begin{abstract}
Let $R$ be a commutative noetherian ring with a dualizing complex. By recent work of Iyengar and Krause (2006), the difference between the category of acyclic complexes and its subcategory of totally acyclic complexes measures how far $R$ is from being Gorenstein. In particular, $R$ is Gorenstein if and only if every acyclic complex is totally acyclic.

In this note we exhibit a specific acyclic complex with the property that it is totally acyclic if and only if $R$ is Gorenstein.
\end{abstract}

\section{INTRODUCTION}

Let $R$ be a commutative noetherian ring. A complex $X$ of $R$-modules is said to be acyclic if it has zero homology, i.e. $\mathrm{H}(X)=0$. An acyclic complex of projective modules is called totally acyclic if the acyclicity is preserved by $\operatorname{Hom}_{R}(-, P)$ for every projective module $P$. Dually, an acyclic complex of injective modules is totally acyclic if the acyclicity is preserved by $\operatorname{Hom}_{R}(I,-)$ for every injective module $I$.

Over a Gorenstein ring, every acyclic complex of projective or of injective modules is totally acyclic. Iyengar and Krause have recently proved a converse; indeed, by $[9$, cor. 5.5$]$ the following are equivalent when $R$ has a dualizing complex:

(i) The ring $R$ is Gorenstein.

(ii) Every acyclic complex of projective $R$-modules is totally acyclic.

(iii) Every acyclic complex of injective $R$-modules is totally acyclic.

Moreover, for a local ring $(R, \mathfrak{m})$ that is not Gorenstein and has $\mathfrak{m}^{2}=0$ there is a natural example, provided by [9, prop. 6.1(3)], of an acyclic, but not totally acyclic, complex of projective $R$-modules.

The purpose of this note is to prove that for every ring $R$ with a dualizing complex $D$, a specific acyclic complex $K$, defined in 2.1, serves as a test complex for Gorensteinness in the following sense: The $\operatorname{ring} R$ is Gorenstein if and only if $K \otimes_{R} D$ is acyclic. This is achieved by Theorem 2.2. In general, $K$ is an acyclic complex of flat $R$-modules. Corollary 2.6 shows that if $R$ is an artinian local ring, then $K$ is a complex of projective modules, and (i)-(iii) above are equivalent with

(iv) The complex $K$ is totally acyclic.

Received by the editors July 14, 2006 and, in revised form, December 6, 2006 and January 17, 2007.

2000 Mathematics Subject Classification. Primary 13H10, 13D25.

Key words and phrases. Gorenstein rings, dualizing complexes, totally acyclic complexes.

The first author was partly supported by a grant from the Carlsberg Foundation. 
Test complexes of injective modules can be obtained directly from $K$ (Corollary 2.5 ) or through a potentially different construction explored in Section 3. The authors of [9] have pointed out that the latter is of particular interest, as it yields a generator for $\mathbf{K}_{\mathrm{ac}}(\operatorname{Inj} \mathrm{R}) / \mathbf{K}_{\mathrm{tac}}(\operatorname{Inj} \mathrm{R})$, the Verdier quotient of acyclic complexes modulo totally acyclic complexes in the homotopy category of injective $R$-modules. This is proved in Theorem 3.5.

\section{BACKGROUND}

Throughout this paper $R$ is a commutative noetherian ring. The notation $(R, \mathfrak{m}, k)$ means $R$ is local with maximal ideal $\mathfrak{m}$ and residue field $k$.

Complexes of $R$-modules ( $R$-complexes for short) are graded homologically,

$$
X=\cdots \rightarrow X_{i+1} \stackrel{\partial_{i+1}^{X}}{\longrightarrow} X_{i} \stackrel{\partial_{i}^{X}}{\longrightarrow} X_{i-1} \rightarrow \cdots .
$$

The suspension of $X$ is denoted $\Sigma X$; it is the complex with $(\Sigma X)_{i}=X_{i-1}$ and differential $\partial^{\Sigma X}=-\partial^{X}$. A complex $X$ is said to be bounded if $X_{i}=0$ for $|i| \gg 0$.

An isomorphism between $R$-complexes is denoted by ' $\cong$ '; we write $X \cong Y$ if there exists an isomorphism $X \stackrel{\cong}{\longrightarrow} Y$.

A morphism between $R$-complexes is called a quasi-isomorphism, and denoted $X \stackrel{\simeq}{\longrightarrow} Y$ if the induced map in homology, $\mathrm{H}(X) \rightarrow \mathrm{H}(Y)$, is an isomorphism. Following [1, sec. 1] we write $X \simeq Y$, if $X$ and $Y$ can be linked by a sequence of quasi-isomorphisms with arrows in alternating directions. Recall that a morphism $X \rightarrow Y$ is a quasi-isomorphism if and only if its mapping cone, written Cone $(X \rightarrow Y)$, is acyclic.

1.1. Resolutions. The following facts are established in $[1, \text { sec. } 1]^{1}$ and $[2]$.

Every $R$-complex $X$ has a semi-projective resolution. That is, there is a quasiisomorphism $P \stackrel{\simeq}{\longrightarrow} X$, where $P$ is a complex of projective $R$-modules such that $\operatorname{Hom}_{R}(P,-)$ preserves quasi-isomorphisms. For such a complex, also the functor $-\otimes_{R} P$ preserves quasi-isomorphisms. In particular, for any $R$-complexes $Y \simeq Z$ we have $\operatorname{Hom}_{R}(P, Y) \simeq \operatorname{Hom}_{R}(P, Z)$ and $Y \otimes_{R} P \simeq Z \otimes_{R} P$.

If there is an $l$ such that $\mathrm{H}_{i}(X)=0$ for $i<l$, then $X$ has a semi-projective resolution $P$ with $P_{i}=0$ for $i<l$. If, in addition, $\mathrm{H}_{i}(X)$ is finitely generated for all $i$, then $P$ can be chosen with all modules $P_{i}$ finitely generated.

Every $R$-complex $X$ has a semi-injective resolution. That is, there is a quasiisomorphism $X \stackrel{\simeq}{\longrightarrow} J$, where $J$ is a complex of injective $R$-modules such that $\operatorname{Hom}_{R}(-, J)$ preserves quasi-isomorphisms. In particular, for such a complex $J$ and any $R$-complexes $Y \simeq Z$ we have $\operatorname{Hom}_{R}(Y, J) \simeq \operatorname{Hom}_{R}(Z, J)$.

1.2. Lemma. Let $X$ and $Y$ be $R$-complexes such that either $X_{i}=0$ for all $i \ll 0$ or $Y_{i}=0$ for all $i \gg 0$. If $\mathrm{H}\left(X_{i} \otimes_{R} Y\right)=0$ for all $i \in \mathbb{Z}$, then $\mathrm{H}\left(X \otimes_{R} Y\right)=0$.

Proof. Let $E$ be a faithfully injective $R$-module. The complex $X \otimes_{R} Y$ is acyclic if and only if $\operatorname{Hom}_{R}\left(X \otimes_{R} Y, E\right) \cong \operatorname{Hom}_{R}\left(X, \operatorname{Hom}_{R}(Y, E)\right)$ is so. The claim is now immediate from [5, lem. (2.4)].

\footnotetext{
${ }^{1}$ Where semi-projective/injective resolutions are called DG-projective/injective.
} 
1.3. Dualizing complexes. Following [8, V.§2], a dualizing complex for $R$ is a bounded complex $D$ of injective $R$-modules such that $\mathrm{H}_{i}(D)$ is finitely generated for all $i \in \mathbb{Z}$, and the homothety morphism

$$
\chi^{D}: R \longrightarrow \operatorname{Hom}_{R}(D, D)
$$

is a quasi-isomorphism.

Let $(R, \mathfrak{m}, k)$ be a local ring with a dualizing complex $D$. After suspensions we can assume $D$ is normalized, cf. [8, V. $\S 5]$, in which case [8, prop. V.3.4] yields

$$
\mathrm{H}\left(\operatorname{Hom}_{R}(k, D)\right) \cong k \text {. }
$$

If $R$ is artinian, then $\mathrm{E}_{R}(k)$, the injective hull of the residue field, is a normalized dualizing complex for $R$.

\section{A TEST COMPLEX OF FLAT MODULES}

2.1. A distinguished complex of flat modules. Assume that $R$ has a dualizing complex $D$, and let $\pi: P \stackrel{\simeq}{\longrightarrow} D$ be a semi-projective resolution. By 1.1 we can assume that $P$ consists of finitely generated modules with $P_{i}=0$ for all $i \ll 0$. The functors $\operatorname{Hom}_{R}(P,-)$ and $\operatorname{Hom}_{R}(-, D)$ preserve quasi-isomorphisms, so the commutative diagram

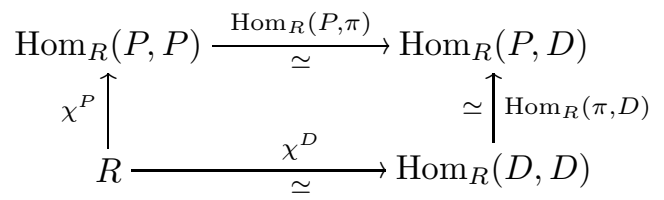

shows that the homothety map $\chi^{P}$ is a quasi-isomorphism. In particular,

$$
K=\text { Cone }\left(R \stackrel{\chi^{P}}{\longrightarrow} \operatorname{Hom}_{R}(P, P)\right)
$$

is acyclic. The modules in $\operatorname{Hom}_{R}(P, P)$ are direct products of modules of the form $\operatorname{Hom}_{R}\left(P_{i}, P_{i+n}\right)$, and each such module is flat. Thus, $\chi^{P}$ is a quasi-isomorphism between complexes of flat $R$-modules, and the mapping cone $K$ is, therefore, an acyclic complex of flat $R$-modules.

We can now state the main result; the proof is given at the end of the section.

2.2. Theorem. Let $R$ be a commutative noetherian ring with a dualizing complex $D$, and let $K$ be the acyclic complex of flat modules defined in 2.1. The ring $R$ is Gorenstein if and only if the complex $K \otimes_{R} D$ is acyclic.

2.3. Remark. While $C=$ Cone $\chi^{D}$ is also an acyclic complex of flat $R$-modules, it cannot detect Gorensteinness. Indeed, $C$ is bounded, so $C \otimes_{R} X$ is acyclic for every $R$-complex $X$ by Lemma 1.2. If $R$ is artinian, then $C$ is even split exact.

2.4. Remark. In the theory of Gorenstein dimensions, there is a notion of a complete flat resolution - due to Enochs, Jenda, and Torrecillas [6] — namely an acyclic complex $F$ of flat modules such that $F \otimes_{R} I$ is acyclic for every injective module $I$.

If $R$ is Gorenstein, then every acyclic complex of flat $R$-modules is a complete flat resolution. Indeed, every injective $R$-module $I$ has finite flat dimension, and then it is straightforward to verify that the functor $-\otimes_{R} I$ preserves acyclicity of complexes of flat modules. On the other hand, let $K$ and $D$ be as in Theorem 2.2. If $K$ is a complete flat resolution, then $K \otimes_{R} D$ is acyclic by Lemma 1.2. 
Thus, the following assertions are equivalent:

(i) The ring $R$ is Gorenstein.

(ii) The complex $K$ is a complete flat resolution.

(iii) Every acyclic complex of flat modules is a complete flat resolution.

The complex $K$ defined in 2.1 appears to be a natural test object for Gorensteinness. However, it might in the context of [9] be of interest to exhibit a test complex of injective or of projective modules.

To this end, we first note that the next corollary to Theorem 2.2 is immediate in view of Remark 2.4 and [3, prop. (6.4.1)]. See Section 3 for a further discussion of test complexes of injective modules.

2.5. Corollary. Let $R$ be a commutative noetherian ring with a dualizing complex. Let $K$ be the acyclic complex of flat modules defined in 2.1, and let $E$ be a faithfully injective $R$-module. The complex $\operatorname{Hom}_{R}(K, E)$ is an acyclic complex of injective modules, and $R$ is Gorenstein if and only if $\operatorname{Hom}_{R}(K, E)$ is totally acyclic.

For artinian local rings $(R, \mathfrak{m})$, Theorem 2.2 provides a test complex of projective modules. In particular, for $R$ with $\mathfrak{m}^{2}=0$ the following recovers [9, prop. 6.1(3)].

2.6. Corollary. Let $R$ be an artinian local ring. The complex $K$ defined in 2.1 is an acyclic complex of projective $R$-modules, and $R$ is Gorenstein if and only if $K$ is totally acyclic.

Proof. When $R$ is artinian and local, every flat $R$-module is projective. Thus, $K$ is an acyclic complex of projective modules.

The "only if" part is well-known. To prove "if", assume $K$ is totally acyclic and recall from 1.3 that the module $E=\mathrm{E}_{R}(k)$ is dualizing for $R$. The first of the following isomorphisms is induced by $\chi^{E}$, and the second is Hom-tensor adjointness

$$
\operatorname{Hom}_{R}(K, R) \cong \operatorname{Hom}_{R}\left(K, \operatorname{Hom}_{R}(E, E)\right) \cong \operatorname{Hom}_{R}\left(K \otimes_{R} E, E\right) .
$$

The complex $\operatorname{Hom}_{R}(K, R)$ is acyclic and $E$ is faithfully injective, so $K \otimes_{R} E$ is acyclic and, therefore, $R$ is Gorenstein by Theorem 2.2.

For the proof of Theorem 2.2 we need a technical lemma.

2.7. Lemma. Let $P$ be an $R$-complex of finitely generated projective modules, $X$ be any $R$-complex, and $B$ be a bounded $R$-complex of finitely generated modules. There is an isomorphism of $R$-complexes

$$
\omega: \operatorname{Hom}_{R}(P, X) \otimes_{R} B \stackrel{\cong}{\longrightarrow} \operatorname{Hom}_{R}\left(P, X \otimes_{R} B\right) \text {. }
$$

Proof. It is straightforward to check that the assignment

$$
\omega(\phi \otimes b)(p)=(-1)^{|p||b|} \phi(p) \otimes b,
$$

where $|\cdot|$ denotes the degree of an element, defines a morphism between the relevant complexes. By assumption, there exist integers $l \leqslant u$ such that $B_{h}=0$ when $h<l$ 
or $h>u$. For every $n \in \mathbb{Z}$ we have

$$
\begin{aligned}
\left(\operatorname{Hom}_{R}(P, X) \otimes_{R} B\right)_{n} & =\bigoplus_{i=n-u}^{n-l} \operatorname{Hom}_{R}(P, X)_{i} \otimes_{R} B_{n-i} \\
& =\bigoplus_{i=n-u}^{n-l}\left(\prod_{j \in \mathbb{Z}} \operatorname{Hom}_{R}\left(P_{j}, X_{j+i}\right)\right) \otimes_{R} B_{n-i} \\
& \cong \bigoplus_{i=n-u}^{n-l} \prod_{j \in \mathbb{Z}}\left(\operatorname{Hom}_{R}\left(P_{j}, X_{j+i}\right) \otimes_{R} B_{n-i}\right) \\
& \cong \bigoplus_{i=n-u}^{n-l} \prod_{j \in \mathbb{Z}} \operatorname{Hom}_{R}\left(P_{j}, X_{j+i} \otimes_{R} B_{n-i}\right) \\
& \cong \prod_{j \in \mathbb{Z}} \operatorname{Hom}_{R}\left(P_{j}, \bigoplus_{i=n-u}^{n-l} X_{j+i} \otimes_{R} B_{n-i}\right) \\
& =\prod_{j \in \mathbb{Z}} \operatorname{Hom}_{R}\left(P_{j},\left(X \otimes_{R} B\right)_{j+n}\right) \\
& =\operatorname{Hom}_{R}\left(P, X \otimes_{R} B\right)_{n} .
\end{aligned}
$$

Since the modules $B_{n-i}$ are finitely generated, the functors $-\otimes_{R} B_{n-i}$ commute with arbitrary products for every $i$; this explains the first isomorphism. The modules $P_{j}$ are finitely generated and projective, so for all $i, j$, and $n$ the homomorphism of modules

$$
\operatorname{Hom}_{R}\left(P_{j}, X_{j+i}\right) \otimes_{R} B_{n-i} \stackrel{\omega_{i j n}}{\longrightarrow} \operatorname{Hom}_{R}\left(P_{j}, X_{j+i} \otimes_{R} B_{n-i}\right)
$$

is invertible, and this accounts for the second isomorphism. Thus, $\omega$ is an isomorphism of graded modules, and the sign in the definition of $\omega$ ensures that it commutes with the differentials.

Proof of Theorem 2.2. The "only if" part was settled in Remark 2.4.

For the "if" part, assume that the complex $K \otimes_{R} D$ is acyclic; the isomorphism Cone $\left(\chi^{P} \otimes_{R} D\right) \cong K \otimes_{R} D$ implies that

$$
\chi^{P} \otimes_{R} D: D \longrightarrow \operatorname{Hom}_{R}(P, P) \otimes_{R} D
$$

is a quasi-isomorphism.

Choose an $n$ such that $\mathrm{H}_{i}(D)=0$ for all $i>n$, and let $B$ be the soft truncation of $P$ on the left at $n$ :

$$
B=0 \longrightarrow \text { Coker } \partial_{n+1}^{P} \stackrel{\overline{\partial_{n}^{P}}}{\longrightarrow} P_{n-1} \stackrel{\partial_{n-1}^{P}}{\longrightarrow} P_{n-2} \longrightarrow \cdots .
$$

There are quasi-isomorphisms $B \stackrel{\simeq}{\longleftarrow} \stackrel{\simeq}{\longrightarrow} D$ and, hence, a quasi-isomorphism $\beta: B \stackrel{\simeq}{\longrightarrow} D$; see $[1,1.1$. I.(1) and 1.4.I]. Since the mapping cone of $\beta$ is a bounded acyclic complex, and $\operatorname{Hom}_{R}(P, P)$ is a complex of flat modules, Lemma 1.2 applies to show that also $\operatorname{Hom}_{R}(P, P) \otimes_{R} \operatorname{Cone}(\beta)$ is acyclic. Thus, the isomorphism $\operatorname{Cone}\left(\operatorname{Hom}_{R}(P, P) \otimes_{R} \beta\right) \cong \operatorname{Hom}_{R}(P, P) \otimes_{R} \operatorname{Cone}(\beta)$ implies that

$$
\operatorname{Hom}_{R}(P, P) \otimes_{R} \beta: \operatorname{Hom}_{R}(P, P) \otimes_{R} B \longrightarrow \operatorname{Hom}_{R}(P, P) \otimes_{R} D
$$

is a quasi-isomorphism. 
By the choice of $P$, cf. 2.1, the bounded complex $B$ consists of finitely generated modules, and Lemma 2.7 yields an isomorphism

$$
\omega: \operatorname{Hom}_{R}(P, P) \otimes_{R} B \stackrel{\cong}{\longrightarrow} \operatorname{Hom}_{R}\left(P, P \otimes_{R} B\right) \text {. }
$$

Finally, let $\iota: P \otimes_{R} B \stackrel{\simeq}{\longrightarrow} J$ be a semi-injective resolution; the quasi-isomorphism $\iota$ is preserved by $\operatorname{Hom}_{R}(P,-)$, and the resulting quasi-isomorphism combined with $(1),(2)$, and (3) yields

$$
D \simeq \operatorname{Hom}_{R}(P, J)
$$

It suffices to prove that $R_{\mathfrak{m}}$ is Gorenstein for every maximal ideal $\mathfrak{m}$ of $R$. Let $\mathfrak{m}$ be a maximal ideal; the complex $D_{\mathfrak{m}}$ is dualizing for $R_{\mathfrak{m}}$; see [8, cor. V.2.3]. Set $k=R_{\mathfrak{m}} / \mathfrak{m} R_{\mathfrak{m}} \cong R / \mathfrak{m}$. We may, after suspensions, assume $D_{\mathfrak{m}}$ is normalized, so $\mathrm{H}\left(\operatorname{Hom}_{R_{\mathfrak{m}}}\left(k, D_{\mathfrak{m}}\right)\right) \cong k$; see (1.3.1). Moreover, there are isomorphisms $\operatorname{Hom}_{R}(k, D) \cong \operatorname{Hom}_{R}(k, D) \otimes_{R} R_{\mathfrak{m}} \cong \operatorname{Hom}_{R_{\mathfrak{m}}}\left(k, D_{\mathfrak{m}}\right)$, so we have

$$
k \cong \mathrm{H}\left(\operatorname{Hom}_{R}(k, D)\right) .
$$

Let $v: Q \stackrel{\simeq}{\longrightarrow} k$ be a semi-projective resolution of $k$ over $R$. As $\operatorname{Hom}_{R}(-, D)$ preserves quasi-isomorphisms, we have

$$
\operatorname{Hom}_{R}(k, D) \stackrel{\simeq}{\longrightarrow} \operatorname{Hom}_{R}(Q, D) .
$$

Also $\operatorname{Hom}_{R}(Q,-)$ preserves quasi-isomorphisms, and from (4) we get

$$
\operatorname{Hom}_{R}(Q, D) \simeq \operatorname{Hom}_{R}\left(Q, \operatorname{Hom}_{R}(P, J)\right) \cong \operatorname{Hom}_{R}\left(Q \otimes_{R} P, J\right),
$$

where the isomorphism is Hom-tensor adjointness. Finally, $v \otimes_{R} P$ is a quasiisomorphism, and hence so is

$$
\operatorname{Hom}_{R}\left(v \otimes_{R} P, J\right): \operatorname{Hom}_{R}\left(k \otimes_{R} P, J\right) \stackrel{\simeq}{\longrightarrow} \operatorname{Hom}_{R}\left(Q \otimes_{R} P, J\right) .
$$

Combining (5)-(8) and again using Hom-tensor adjointness, we obtain

$$
\begin{aligned}
k & \cong \mathrm{H}\left(\operatorname{Hom}_{R}\left(k \otimes_{R} P, J\right)\right) \\
& \cong \mathrm{H}\left(\operatorname{Hom}_{R}\left(\left(k \otimes_{R} P\right) \otimes_{k} k, J\right)\right) \\
& \cong \mathrm{H}\left(\operatorname{Hom}_{k}\left(k \otimes_{R} P, \operatorname{Hom}_{R}(k, J)\right)\right) \\
& \cong \operatorname{Hom}_{k}\left(\mathrm{H}\left(k \otimes_{R} P\right), \mathrm{H}\left(\operatorname{Hom}_{R}(k, J)\right)\right) .
\end{aligned}
$$

Thus, $\operatorname{Hom}_{k}\left(\mathrm{H}\left(k \otimes_{R} P\right), \mathrm{H}\left(\operatorname{Hom}_{R}(k, J)\right)\right)$ is a finitely generated $k$-vector space; in particular, $\mathrm{H}\left(k \otimes_{R} P\right)$ must be finitely generated. Note that $\mathrm{H}_{i}\left(k \otimes_{R} P\right) \cong$ $\mathrm{H}_{i}\left(k \otimes_{R_{\mathfrak{m}}} P_{\mathfrak{m}}\right)$ for all $i \in \mathbb{Z}$; it follows that $\mathrm{H}_{i}\left(k \otimes_{R_{\mathfrak{m}}} P_{\mathfrak{m}}\right)=0$ for all $i \gg 0$. By [1, prop. 5.5] the dualizing $R_{\mathfrak{m}}$-complex $D_{\mathfrak{m}}$ then has finite flat dimension, and hence $R_{\mathfrak{m}}$ is Gorenstein; see [7, thm. (17.23)] or [4, thm. (8.1)].

\section{A test COMPleX OF INJECTIVE MOdules}

The next construction is another source for test complexes. 
3.1. A distinguished complex of injective modules. Assume $R$ has a dualizing complex $D$. As in 2.1, let $\pi: P \stackrel{\simeq}{\longrightarrow} D$ be a semi-projective resolution of $D$ consisting of finitely generated modules with $P_{i}=0$ for all $i \ll 0$. The assignment $\varphi \otimes p \mapsto \varphi(p)$ defines a morphism of complexes, $\varepsilon$, such that the following diagram is commutative:

$$
\begin{aligned}
& \begin{aligned}
\operatorname{Hom}_{R}(P, D) \otimes_{R} P \longrightarrow & \underset{\uparrow}{D} \\
\operatorname{Hom}_{R}(P, \pi) \otimes_{R} P \uparrow \simeq & \simeq \pi
\end{aligned} \\
& \operatorname{Hom}_{R}(P, P) \otimes_{R} P \underset{\chi^{P} \otimes_{R} P}{\simeq} R \otimes_{R} P .
\end{aligned}
$$

Thus, $\varepsilon$ is a quasi-isomorphism between complexes of injective $R$-modules, and the mapping cone

$$
M=\text { Cone }\left(\operatorname{Hom}_{R}(P, D) \otimes_{R} P \stackrel{\varepsilon}{\longrightarrow} D\right)
$$

an acyclic complex of injective $R$-modules.

An argument similar to the proof of Theorem 2.2 yields the next result, which is also a corollary of Theorem 3.5.

3.2. Theorem. Let $R$ be a commutative noetherian ring with a dualizing complex, and let $M$ be the acyclic complex of injective modules defined in 3.1. The ring $R$ is Gorenstein if and only if $M$ is totally acyclic.

3.3. Remark. If $(R, \mathfrak{m}, k)$ is an artinian local ring, then there is an isomorphism

$$
K \cong \Sigma \operatorname{Hom}_{R}\left(M, \mathrm{E}_{R}(k)\right)
$$

where $K$ and $M$ are the complexes from 2.1 and 3.1, and $\mathrm{E}_{R}(k)$ is the injective hull of $k$. Indeed, with $E=\mathrm{E}_{R}(k)$ there is a commutative diagram

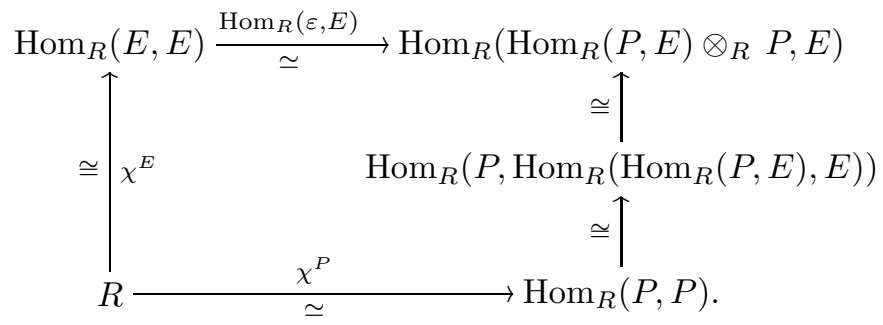

The vertical maps on the right are the natural isomorphisms, and because $E$ is a module, the homothety map $\chi^{E}$ is also a genuine isomorphism. The diagram induces the desired isomorphism between the complexes $K=$ Cone $\left(\chi^{P}\right)$ and Cone $\left(\operatorname{Hom}_{R}(\varepsilon, E)\right) \cong \Sigma \operatorname{Hom}_{R}(\operatorname{Cone}(\varepsilon), E)=\Sigma \operatorname{Hom}_{R}(M, E)$.

When $R$ is not artinian, we do not know if the complexes $K$ and $M$ are related.

Using [5, prop. (5.1)] it is not hard to prove the next parallel to Corollary 2.5.

3.4. Corollary. Let $R$ be a commutative noetherian ring with a dualizing complex. Let $M$ be the acyclic complex of injective modules defined in 3.1, and let $E$ be a faithfully injective $R$-module. The complex $\operatorname{Hom}_{R}(M, E)$ is an acyclic complex of flat modules, and $R$ is Gorenstein if and only if $\operatorname{Hom}_{R}(M, E) \otimes_{R} I$ is acyclic for every injective module $I$.

In conversations, the authors of [9] have informed us of Theorem 3.5 below; note that it contains Theorem 3.2. For notation and terminology we refer to [9]. 
3.5. Theorem. Let $R$ be a commutative noetherian ring with a dualizing complex. The acyclic complex $M$ of injective modules defined in 3.1 generates the quotient category $\mathbf{K}_{\mathrm{ac}}(\operatorname{Inj} \mathrm{R}) / \mathbf{K}_{\mathrm{tac}}(\operatorname{Inj} \mathrm{R})$.

Proof. By $\left[9,1.7,5.4\right.$, and 5.9(3)] the quotient category $\mathbf{K}_{\mathrm{ac}}(\operatorname{Inj} \mathrm{R}) / \mathbf{K}_{\mathrm{tac}}(\operatorname{Inj} \mathrm{R})$ is generated by the image of the dualizing complex $D$ under the equivalence $\mathbf{D}^{f}(R) \stackrel{\sim}{\longrightarrow}$ $\mathbf{K}^{\mathrm{c}}(\operatorname{Inj} \mathrm{R}) ;$ cf. [9, 2.3(2)].

Let $P \stackrel{\simeq}{\longrightarrow} D$ be a semi-projective resolution. The functor $\operatorname{Hom}_{R}(P,-)$ preserves quasi-isomorphisms, so the composite

$$
R \stackrel{\simeq}{\longrightarrow} \operatorname{Hom}_{R}(P, P) \stackrel{\simeq}{\longrightarrow} \operatorname{Hom}_{R}(P, D)
$$

provides an injective resolution $R \stackrel{\simeq}{\longrightarrow} \mathrm{i} R=\operatorname{Hom}_{R}(P, D)$. Since $\mathrm{i} R$ is a compact object in $\mathbf{K}(\operatorname{Inj} \mathrm{R})$, the inclusion of the localizing subcategory $\operatorname{Loc}(\mathrm{i} R) \subseteq \mathbf{K}(\operatorname{Inj} \mathrm{R})$ admits a right adjoint $\rho: \mathbf{K}(\operatorname{Inj} \mathrm{R}) \rightarrow \operatorname{Loc}(\mathrm{i} R)$; see $[9,1.5 .1]$. By $[9,2.3(2)]$ the image of $D$ in $\mathbf{K}(\operatorname{Inj} R)$ is

$$
\operatorname{Cone}(\rho(D) \stackrel{\xi}{\rightarrow} D)
$$

where $\xi$ is the natural map.

It remains to show that $M \cong \operatorname{Cone}(\rho(D) \stackrel{\xi}{\rightarrow} D)$. It suffices to establish a commutative diagram,

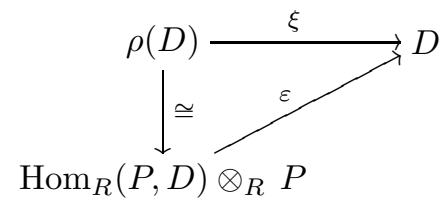

The complex $\operatorname{Hom}_{R}(P, D) \otimes_{R} P=\mathrm{i} R \otimes_{R} P$ is in Loc $(\mathrm{i} R)$, and since $\varepsilon$ is a quasiisomorphism, $\operatorname{Hom}_{\mathbf{K}(\operatorname{Inj} \mathrm{R})}(\mathrm{i} R, \varepsilon)$ is an isomorphism; cf. [9, 2.2]. The existence of the desired isomorphism $\rho(D) \cong \operatorname{Hom}_{R}(P, D) \otimes_{R} P$ now follows from $[9,1.4]$.

\section{ACKNOWLEDGMENTS}

It is a pleasure to thank Srikanth Iyengar and Henning Krause for discussions regarding this work and, in particular, for letting us include Theorem 3.5. Thanks are also due to Paul Roberts for suggestions that have improved the exposition of this paper.

\section{REFERENCES}

[1] Luchezar L. Avramov and Hans-Bjørn Foxby, Homological dimensions of unbounded complexes, J. Pure Appl. Algebra 71 (1991), no. 2-3, 129-155. MR1117631 (93g:18017)

[2] Luchezar L. Avramov, Hans-Bjørn Foxby, and Stephen Halperin, Differential graded homological algebra, preprint (2004).

[3] Lars Winther Christensen, Gorenstein dimensions, Lecture Notes in Mathematics, vol. 1747, Springer-Verlag, Berlin, 2000. MR2002e:13032

[4] _ Semi-dualizing complexes and their Auslander categories, Trans. Amer. Math. Soc. 353 (2001), no. 5, 1839-1883 (electronic). MR2002a:13017

[5] Lars Winther Christensen, Anders Frankild, and Henrik Holm, On Gorenstein projective, injective and flat dimensions - A functorial description with applications, J. Algebra 302 (2006), 231-279. MR2236602

[6] Edgar E. Enochs, Overtoun M. G. Jenda, and Blas Torrecillas, Gorenstein flat modules, Nanjing Daxue Xuebao Shuxue Bannian Kan 10 (1993), no. 1, 1-9. MR95a:16004

[7] Hans-Bjørn Foxby, Hyperhomological algebra \& commutative rings, notes in preparation. 
[8] Robin Hartshorne, Residues and duality, Lecture notes of a seminar on the work of A. Grothendieck, given at Harvard 1963/64. With an appendix by P. Deligne. Lecture Notes in Mathematics, No. 20, Springer-Verlag, Berlin, 1966. MR36:5145

[9] Srikanth Iyengar and Henning Krause, Acyclicity versus total acyclicity for complexes over noetherian rings, Doc. Math. 11 (2006), 207-240 (electronic). MR2262932 (2007h:16013)

Department of Mathematics, University of Nebraska, Lincoln, Nebraska 68588 Current address: Department of Mathematics and Statistics, Texas Tech University, Lubbock, Texas 79409

E-mail address: winther@math.unl.edu, lars.w.christensen@ttu.edu

Department of Mathematics, University of Utah, Salt Lake City, Utah 84112

E-mail address: oveliche@math.utah.edu 